\title{
Fabrication of EuF3-Mesocrystals in a gel matrix
}

\begin{tabular}{|c|c|}
\hline Journal: & Zeitschrift für Anorganische und Allgemeine Chemie \\
\hline Manuscript ID: & zaac.201000098.R1 \\
\hline Wiley - Manuscript type: & Article \\
\hline $\begin{array}{r}\text { Date Submitted by the } \\
\text { Author: }\end{array}$ & 18-May-2010 \\
\hline Complete List of Authors: & $\begin{array}{l}\text { Lausser, Christine } \\
\text { Kumke, Michael; University of Potsdam, Institute of Chemistry } \\
\text { (Physical Chemistry) } \\
\text { Antonietti, Markus } \\
\text { Cölfen, Helmut; MPI of Colloids and Interfaces, Colloid Chemistry }\end{array}$ \\
\hline Keywords: & Mesocrystal, Non Classical Crystallization \\
\hline
\end{tabular}

\section{(s) ScholaroNE \\ Manuscript Central}




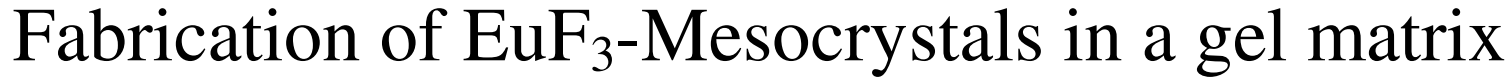

\author{
Christine Lausser ${ }^{1}$, Michael U. Kumke ${ }^{2}$, Markus Antonietti ${ }^{1}$, Helmut Cölfen ${ }^{3} *$ \\ ${ }^{1}$ Max-Planck-Institute of Colloids and Interfaces, Colloid Chemistry, Research Campus \\ Golm, Am Mühlenberg, D-14424 Potsdam \\ ${ }^{2}$ University of Potsdam, Institute of Chemistry (Physical Chemistry), Karl-Liebknecht-Str. 24- \\ 25, 14476 Potsdam, Germany \\ ${ }^{3}$ Present adress: University of Konstanz, Physical Chemistry, Universitätsstr. 10, D-78464 \\ Konstanz, Germany \\ Email: Coelfen@mpikg.mpg.de, helmut.coelfen@uni-konstanz.de
}

Dedicated to the $65^{\text {th }}$ birthday of Rüdiger Kniep, a pioneer of matrix assisted morphosynthesis

\begin{abstract}
Europium(III)fluoride-mesocrystals were synthesised in an organic matrix. This matrix is a gel formed by $\mathrm{Eu}^{3+}$-ions and a polycarboxylate/sulfonate copolymer, ACUSOL 588G. In the gel phase, the local amount of Europium-ions is very high since $\mathrm{Eu}^{3+}$ acts as a crosslinker, and crystallization occurs upon addition of $\mathrm{F}^{-}$. Nucleated seed crystals in the gel phase grow by further ion attachment and form mesocrystals by mutual orientation of the $\mathrm{EuF}_{3}$-particles in the gel. We propose a dipole field as reason for this alignment and that the dipolar character of the particles originates from adsorption of the polyelectrolyte on charged crystal faces.
\end{abstract}

\section{INTRODUCTION}

The control over the mutual self-organization of nanoparticles towards superstructures and well organized arrays is a next step in nanosciences. Recently, non classical crystallization was found to be a tool for controlled nanoparticle organization to superstructures or single crystals, thus generating so-called mesocrystals, superstructures built up by nanocrystals which are in mutual alignment ${ }^{[1-3]}$. Often the crystalline building blocks are in perfect three- 
dimensional crystalline registry. The properties of mesocrystal materials, such as single crystalline-behaviour, high crystallinity and high porosity are very useful for functional materials. Such combination of properties in a single material is unique compared to singlecrystalline, polycrystalline and amorphous materials. A number of desirable characteristics can be found within a mesocrystal that cannot be obtained for the same material in amorphous or single-crystalline form or as a polycrystalline aggregate. For example, it can be very advantageous to use a catalyst-material in form of a mesocrystal. It combines high crystallinity with small particle size, high surface area and high porosity as well as good handling as the mesocrystal has a size in the micrometer range. Examples for mesocrystals of functional materials are $\mathrm{NH}_{4} \mathrm{TiOF}_{3}$ mesocrystals and their topotactic conversion to Anatasemesocrystals ${ }^{[4,5]}$ as well as superparamagnetic Magnetite mesocrystals ${ }^{[6]}$.

In the present study, mesocrystals of Europium(III)fluoride $\left(\mathrm{EuF}_{3}\right)$ are described. Compounds based on rare-earth elements are among the most common luminescent materials, due to high photochemical stability, characteristic sharp emission lines of lanthanide ions and high quantum efficiencies $^{[7]}$. They are applied for high-performance luminescent displays, optical communication and biochemical probes to laser materials ${ }^{[8-11]}$. Therefore, it is desirable to develop bottom up strategies for the design of these materials with a controlled nanostructure. Wang et al prepared a variety of $\mathrm{EuF}_{3}$ nanostructures with different crystalline phases and morphologies via a simple solution route employing different fluoride sources ${ }^{[12,13]}$, and new morphologies of crystalline Europium(III)fluoride were addressed more and more often in the last few years ${ }^{[14,15]}$. The optical properties of $\mathrm{EuF}_{3}$ were analyzed in detail by Jayasankar using fluorescence microscopy. He detected the typical magnetic dipole transition $\left({ }^{5} \mathrm{D}_{0} \rightarrow{ }^{7} \mathrm{~F}_{1}\right)$ and the typical electric dipole transitions $\left({ }^{5} \mathrm{D}_{0} \rightarrow{ }^{7} \mathrm{~F}_{2},{ }^{5} \mathrm{D}_{0} \rightarrow{ }^{7} \mathrm{~F}_{3},{ }^{5} \mathrm{D}_{0} \rightarrow{ }^{7} \mathrm{~F}_{4}\right){ }^{[16]}$.

Following the pioneering work of Kniep on oriented superstructure synthesis in structured biopolymers, e.g. synthesis and analysis of a hexagonal-prismatic seed crystal of fluorapatite formed in a gelatin gel ${ }^{[17,18]}$, we made the current mesocrystals of $\mathrm{EuF}_{3}$ by using a polymer 


\section{RESULTS AND DISCUSSIONS}

As soon as $\mathrm{Eu}\left(\mathrm{NO}_{3}\right)_{3} \cdot 5 \mathrm{H}_{2} \mathrm{O}$ solution is added to the Accusol / $\mathrm{NaBF}_{4}$ solution, nanoparticles composed of polymer and mineral precursors are formed in solution. The particle size is increasing in the first $3 \mathrm{~h}$ to a value of about $500 \mathrm{~nm}$ and then drops because of the sedimentation of the formed particles when they grew further (Figure 1). This is also expressed in the decrease of the intensity, which reaches 0 after 8 hours.
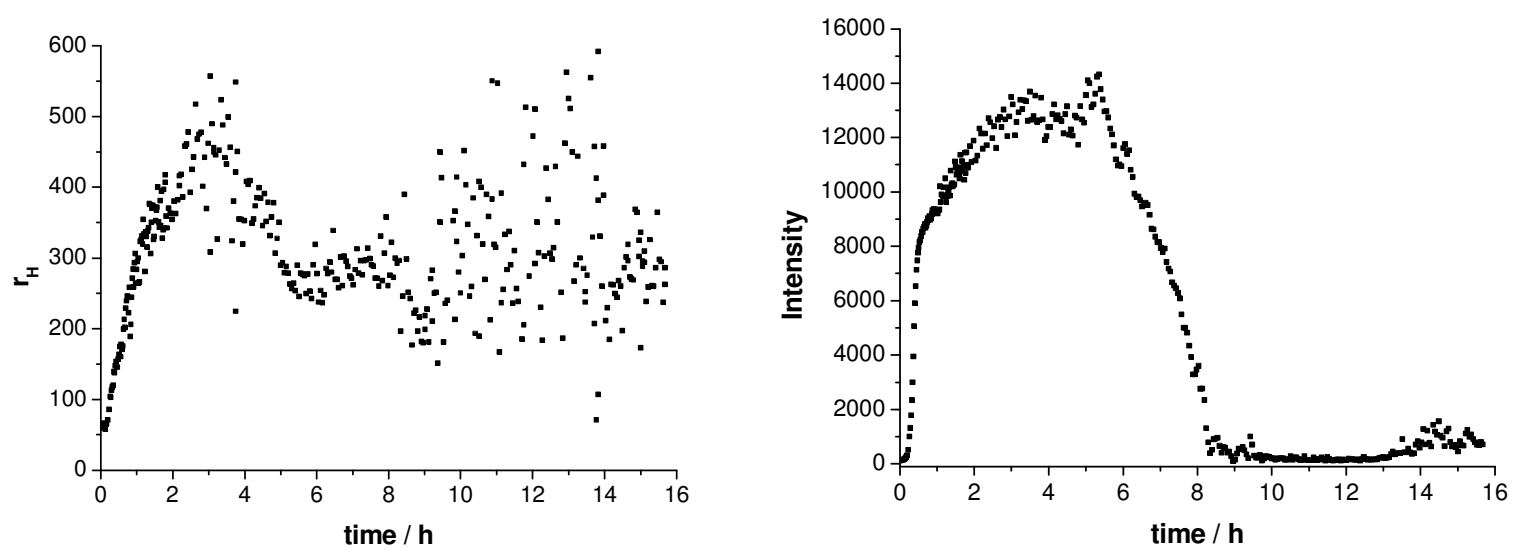

Figure 1: Time dependent dynamic light scattering data of forming $\mathrm{EuF}_{3}$ in presence of Accusol. Left:

Hydrodynamic radius $\mathbf{r}_{H}$ and right: Intensity 
After a reaction time of 3 hours and isolation of crystals, platelet-like agglomerates with flat surfaces, expressed angularity, and with different sizes and thicknesses were obtained. They are obviously built up by small primary nanoparticles with an average size between 30 and 50 nm, as evidenced by SEM (Figure 2). XRD measurements (Figure 3) show that the primary particles are completely crystalline; the diffraction peaks can be indexed to the hexagonal phase of $\mathrm{EuF}_{3}$ (JCPDS 32-0373). The particle size of $30 \mathrm{~nm}$ from Scherrer evaluation agrees well with the SEM results. Comparison of the obtained WAXS data with the reference for hexagonal $\mathrm{EuF}_{3}$ shows that the peak intensities are very similar. No preferred orientation can be deduced from the WAXS data.
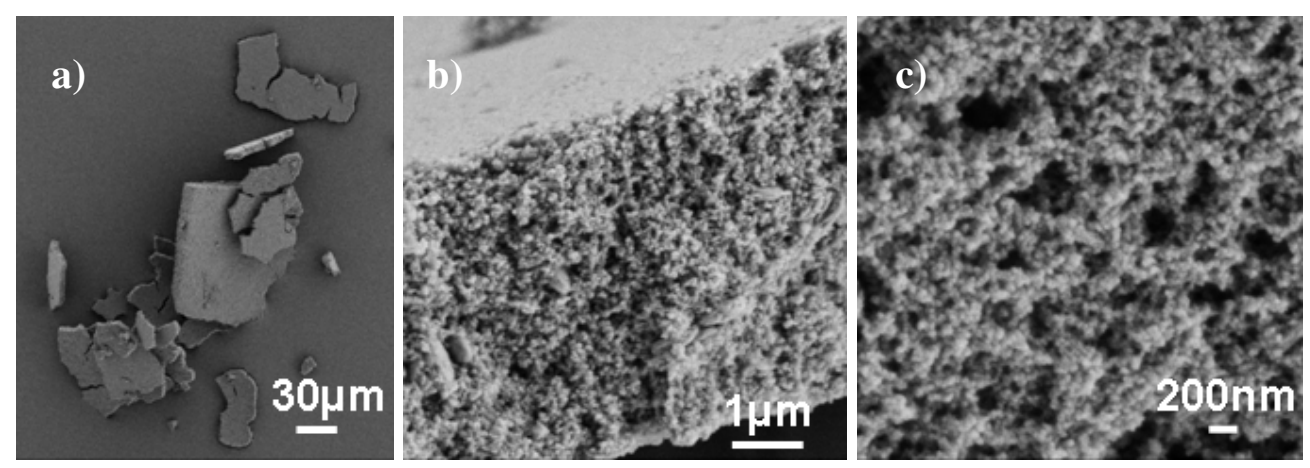

Figure 2: SEM images after a reaction time of 3 hours. a) Agglomerates different in size and form occur. b) and c) the agglomerates are built up by small particles with an average size of $50 \mathrm{~nm}$. 


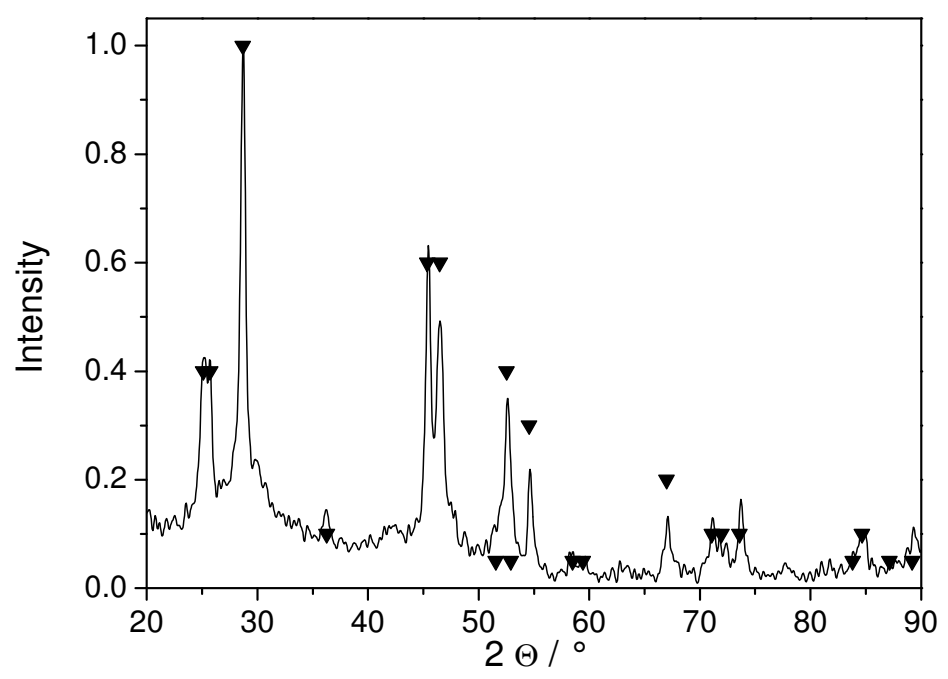

Figure 3: 1: XRD pattern of the sample isolated after a reaction time of 3 hours. The primary crystallite size was estimated to be $30 \mathrm{~nm}$ via the Scherrer-equation. 2: The reference pattern of hexagonal $\mathrm{EuF}_{3}$ obtained from the JCPDS file 32-0373. (triangles)

Nevertheless, the crystalline building blocks of the agglomerates are in perfect vectorial alignment with their three crystallographic axes pointing in joint directions. The electron diffraction on the big polycrystalline aggregates (Figure 4b) gives a single-crystal spot diffraction pattern, which demonstrates the very high degree of mutual orientational order of the nanoparticles. 

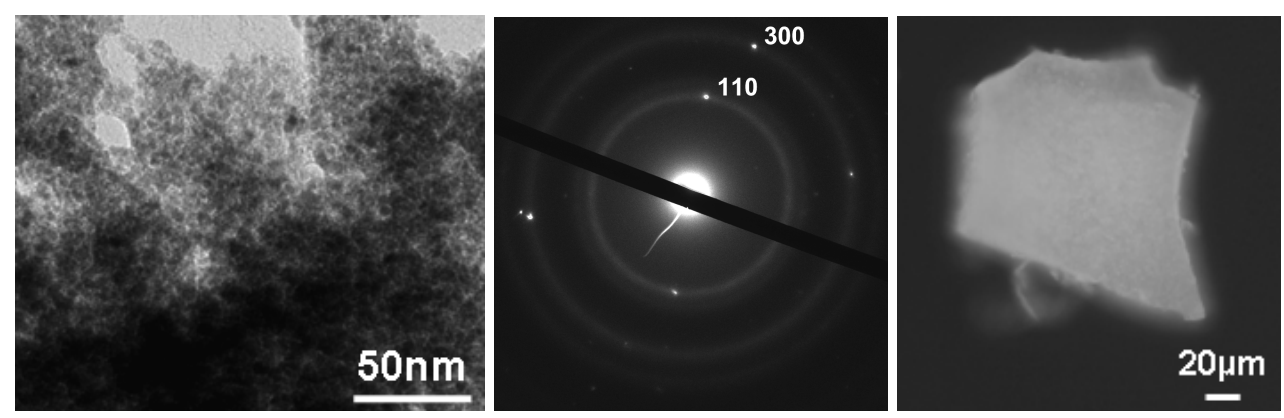

Figure 4: a) TEM image of the sample after a reaction time of 3 hours. It is obvious that the agglomerates are built up by small particles. b) The electron diffraction shows diffraction spots indicating a high mutual order in the agglomerates. c) Polarisation microscope image with crossed polarisers showing the long range order of the particles inside the sample. (see figure SI-1 for a colour image).

In addition, polarisation microscopy with crossed polarisers shows a strong, very homogeneous birefringence with uniform colour over distances $>100 \mu \mathrm{m}$, which is a proof for the iso-orientation of the nanoparticle aggregates even on the micron scale. Therefore, the obtained nanoparticle superstructure is a mesocrystal ${ }^{[1],[2],[19]}$.

The formation reaction of $\mathrm{EuF}_{3}$ is literature described as follows ${ }^{[15]}$

$$
\begin{gathered}
\mathrm{Eu}^{3+}+3 \mathrm{BF}_{4}^{-} \rightarrow \mathrm{EuF}_{3}+3 \mathrm{BF}_{3} \\
\mathrm{BF}_{3}+3 \mathrm{H}_{2} \mathrm{O} \rightarrow 3 \mathrm{H}^{+} 3 \mathrm{~F}^{-}+\mathrm{H}_{3} \mathrm{BO}_{3} \\
\mathrm{Eu}^{3+}+3 \mathrm{~F}^{-} \rightarrow \mathrm{EuF}_{3}
\end{gathered}
$$

The release of the protons (equation 2) and thus the formation of $\mathrm{EuF}_{3}$ can therefore be followed via $\mathrm{pH}$-measurements. The $\mathrm{pH}$ value varied in the experiment from 2.6 at the beginning of the reaction to 2.2 after reaction of 3 hours. This indicates continuous formation of $\mathrm{EuF}_{3}$.

At these $\mathrm{pH}$ values, the carboxylic acid part of the polymer template is clearly protonated, but the strongly acidic sulfonic acid residues (14.7 mol-\%) are still deprotonated. As a consequence, a gel is formed via electrostatic interactions of the sulfonated copolymer with 
$\mathrm{Eu}^{3+} . \mathrm{Gel}$ formation was demonstrated by swelling experiments under a light microscope and gravimetric comparison of the swollen and deswollen gel.

To investigate a possible templating mechanism of the Accusol-Eu gel, we performed a control experiment. By slowly adding an aqueous $\mathrm{Eu}\left(\mathrm{NO}_{3}\right)_{3}$ solution to an aqueous ACUSOL 588G solution, the gel was formed. The mixture became turbid immediately, and colloidal gel particles were formed. The precipitate was centrifuged. SEM images of dried gel samples showed a primary nanostructure similar to the final Europium(III)fluoride-mesocrystals. It is built up by small particles with similar average size of $50 \mathrm{~nm}$ as the building units of a layered gel film (Figure 5). This confirms the assumption that the microgel particles serve as templates for the subsequent crystallisation. The electron diffraction of the control gel particles demonstrates the amorphous character of the polymer-metal ion adducts.

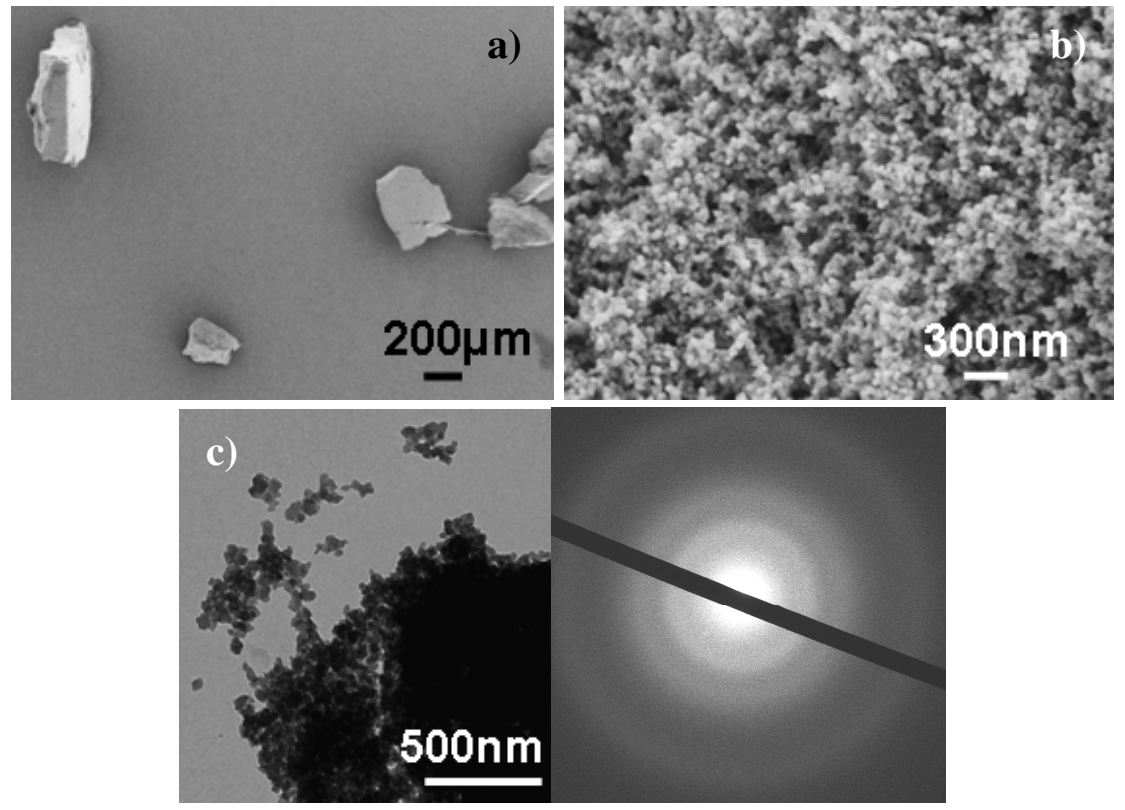

Figure 5: a) -b) SEM-images of the Eu-ACUSOL-gel. c) The TEM-measurement and electron diffraction of the amorphous gel, which is built up by small particles.

Upon addition of $\mathrm{NaBF}_{4}$ aqueous solution to the as formed reference particles, the gel dissolved after three days. After further two days, a transformation to a crystalline precipitate took place and could clearly be followed by naked eye. This shows that the Europium-ions which were initially bound by the polymer reacted with the fluoroborate-ions weakening the 
gel structure, while $\mathrm{EuF}_{3}$ formed. As only those $\mathrm{Eu}^{3+}$ ions, which crosslinked the gel structure, were available for $\mathrm{EuF}_{3}$ formation, the gel was destroyed, and no templating took place in the reference experiment. Therefore, under the standard reaction conditions, an $\mathrm{Eu}^{3+}$ excess with respect to the amount needed to crosslink the gel was mandatory for mesostructure formation. In another control, the experiment was repeated under the same conditions except the removal of the supernatant reaction solution. In that case the Europium-polymer-complex was not dissolved due to the $\mathrm{Eu}^{3+}$ excess. This shows that the fluoroborate-ions react primarily with the Europium-ions in the solution. However, since light scattering (Figure 1) shows that the particles sediment after $3 \mathrm{~h}$ when they have reached a radius of $500 \mathrm{~nm}$. It is likely that these larger particles are the centers for further growth to the structures several tens of micrometer in size as observed by SEM (Figure 2). The polymer and Europium-ions needed for this growth are supplied from the solution phase, and the gel can act as a template for the formation of the $\mathrm{EuF}_{3}$ mesocrystal.

Increasing the reaction time from 3 hours to 16 hours led to some different structures. Round and disc shaped structures with rough surfaces and an average size of $500 \mathrm{~nm}$ were obtained as it is typical for a reaction of $\mathrm{NaBF}_{4}$ and $\mathrm{Eu}\left(\mathrm{NO}_{3}\right)_{3}$ without any additive (Figure 6a,b) ${ }^{[12]}$. The crystallinity of the particles was proven by XRD (data not shown) and the diffraction peaks can be indexed to the hexagonal phase of $\mathrm{EuF}_{3}$.
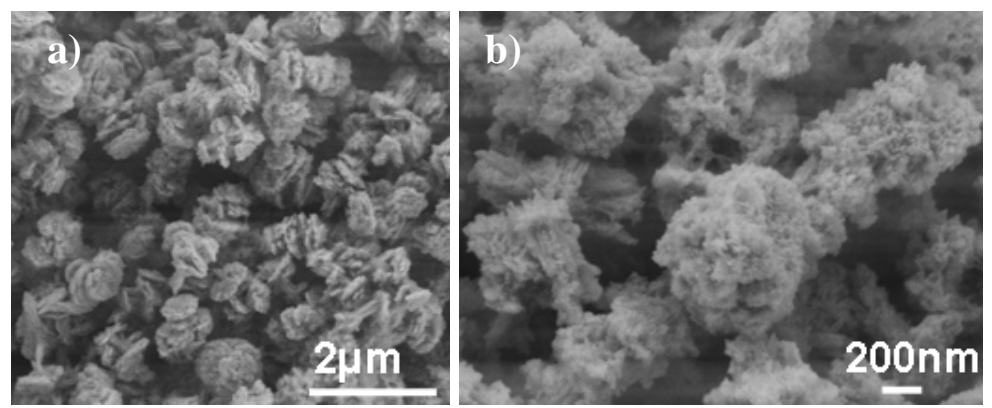


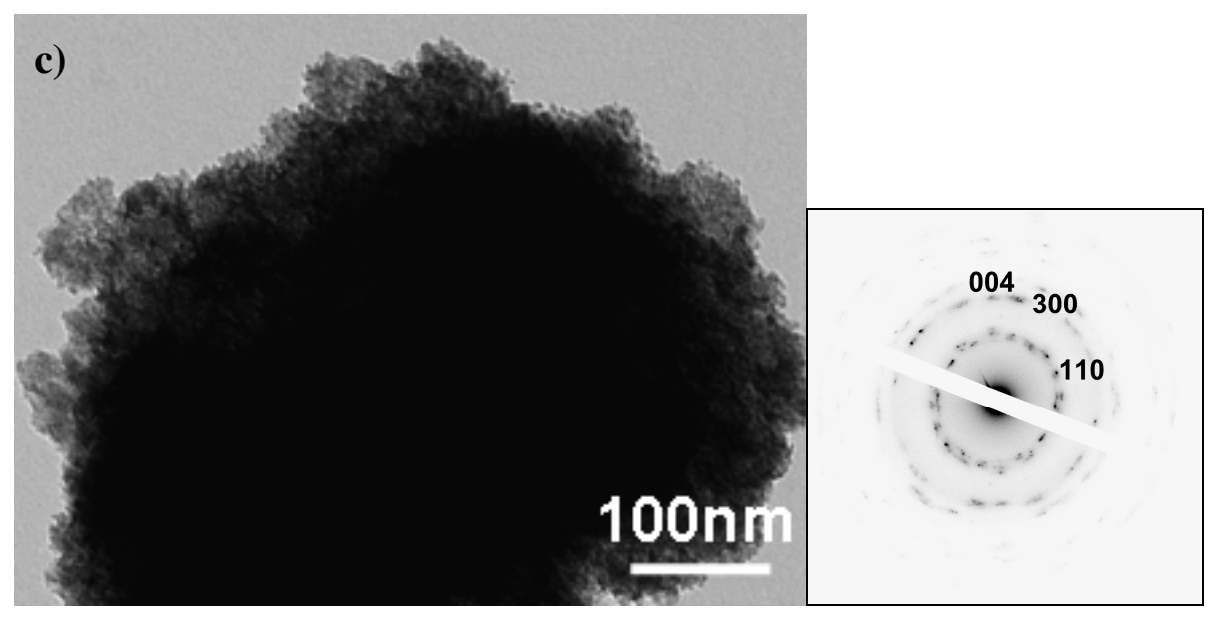

Figure 6: a) and b) The SEM images show a network of round and disc shaped particles after a reaction time of 16h. c) TEM shows that these particles are polycrystalline and exhibit a short range order

These structures are also polycrystalline, and electron diffraction shows again a mutual order as it is typical for mesocrystals (Figure 6c). However, the order is lower than in the case of the mesocrystals formed in the polymer gel template (Figure 4). A time dependent observation of the formed structures $(3 \mathrm{~h}, 5 \mathrm{~h}, 7 \mathrm{~h}, 9 \mathrm{~h}, 11 \mathrm{~h}$ and $16 \mathrm{~h})$ shows that the gel structure controls structure formation up to $7 \mathrm{~h}$ (Figure SI 2). After 9h, the particles with the rough surface begin to form on cost of the polymer gel since the gel gets depleted in the areas where particles are observed (Figure SI 1). After 16h, this process has advanced to a stage, where the entire gel was consumed and only the secondary species were left. We assume that with increasing time $\mathrm{Eu}^{3+}$ gets depleted, and also the $\mathrm{Eu}^{3+}$ crosslinking the polymer is consumed. This leads to the observed dissolution of the gel structure and formation of the round and disk shaped mesocrystals.

The influence of reaction time on the sample properties is also found in the luminescence properties. In Figure 7 the luminescence spectra of the samples (reaction time $3 \mathrm{~h}$ and $16 \mathrm{~h}$ ) and of a reference $\mathrm{EuF}_{3}$ sample are compared. 


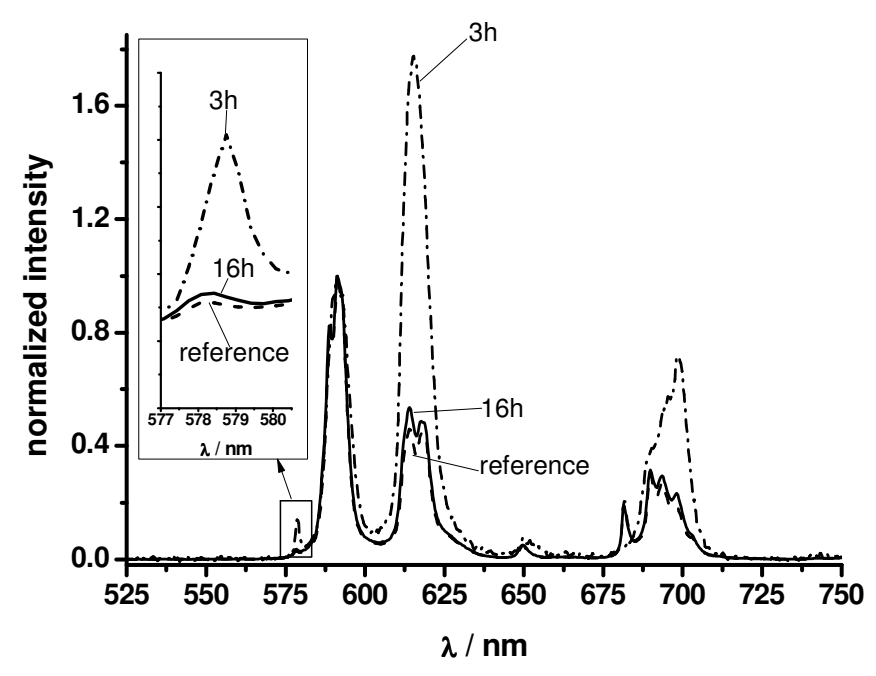

Figure 7 Comparison of the Eu-luminescence spectra of the samples at $3 \mathrm{~h}$ and $16 \mathrm{~h}$. The samples were measured in suspension at room temperature $\left(\lambda_{\mathrm{ex}}=395 \mathrm{~nm}\right)$ in a time-gate detection mode at $\delta \mathrm{t}=20 \mu \mathrm{s}$ after the laser excitation pulse. Inset: Shown is the ${ }^{5} \mathrm{D}_{0^{-}}{ }^{7} \mathrm{~F}_{0}$ transition of europium for the three samples.

A clear difference in the spectral intensity distribution was observed for the samples isolated after $3 \mathrm{~h}$ and 16h: i) for the sample isolated after $3 \mathrm{~h}$ the luminescence transition corresponding to the ${ }^{5} \mathrm{D}_{0^{-}}{ }^{7} \mathrm{~F}_{0}$ transition is well pronounced in contrast to the sample isolated after $16 \mathrm{~h}$. The luminescence spectrum of the sample isolated after $16 \mathrm{~h}$ is very similar to a $\mathrm{EuF}_{3}$ reference sample. The spectral intensity distribution of the europium luminescence is mainly determined by the symmetry of the local coordination sphere and the charge density as well as the covalency of the bonds. While in a highly symmetric complex the ${ }^{5} \mathrm{D}_{0}{ }^{-} \mathrm{F}_{0}$ transition (around $\lambda_{\mathrm{em}}=579 \mathrm{~nm}$ ) is forbidden and therefore should not be observed in the luminescence spectrum, distortion of the symmetry lower the forbiddance and subsequently, this transition can be observed in the luminescence spectrum. In case of the reference sample and the sample isolated after $16 \mathrm{~h}$, the intensity of the ${ }^{5} \mathrm{D}_{0^{-}}{ }^{7} \mathrm{~F}_{0}$ transition is very weak, indicating a high degree of symmetry in crystals (the minor intensity may be due to matrix vibrations, which lower the symmetry and consequently also the forbiddance of the ${ }^{5} \mathrm{D}_{0}{ }^{-} \mathrm{F}_{0}$ transition) (see Figure 7 , Inset). For the sample isolated after $3 \mathrm{~h}$ a much higher intensity of the ${ }^{5} \mathrm{D}_{0^{-}}{ }^{7} \mathrm{~F}_{0}$ transition is 
observed indicating a relaxed europium symmetry in the presence of the polymer gel template. A similar trend is also found for the asymmetry ratio (intensity ratio of the ${ }^{5} \mathrm{D}_{0^{-}}{ }^{7} \mathrm{~F}_{1}$ and the so-called hypersensitive ${ }^{5} \mathrm{D}_{0^{-}}{ }^{7} \mathrm{~F}_{2}$ transition at $\lambda_{\mathrm{em}}=595 \mathrm{~nm}$ and $615 \mathrm{~nm}$, respectively, see also Figure 8). Furthermore, the differences in the europium environment are also reflected in the small spectral shift of the non-degenerate ${ }^{5} \mathrm{D}_{0^{-}}{ }^{7} \mathrm{~F}_{0}$ transition (see Inset of Figure 7), which is representative for a europium species. ${ }^{[28]}$

In Figure 8 the time-resolved luminescence decays at $\lambda_{\mathrm{em}}=595 \mathrm{~nm}$ and the asymmetry ratios are shown, which further support the results of the luminescence spectra analysis. While the reference and the sample isolated after $16 \mathrm{~h}$ are very similar, the luminescence decay as well as the time dependence of the asymmetry ratio of the sample isolated after $3 \mathrm{~h}$ are distinctly different: i) the luminescence decays much faster and ii) the asymmetry ratio continuously changes with time after the laser flash (in contrast to the sample isolated after 16h, which approaches a limiting value similar to the reference sample (see right side of Figure 8)).

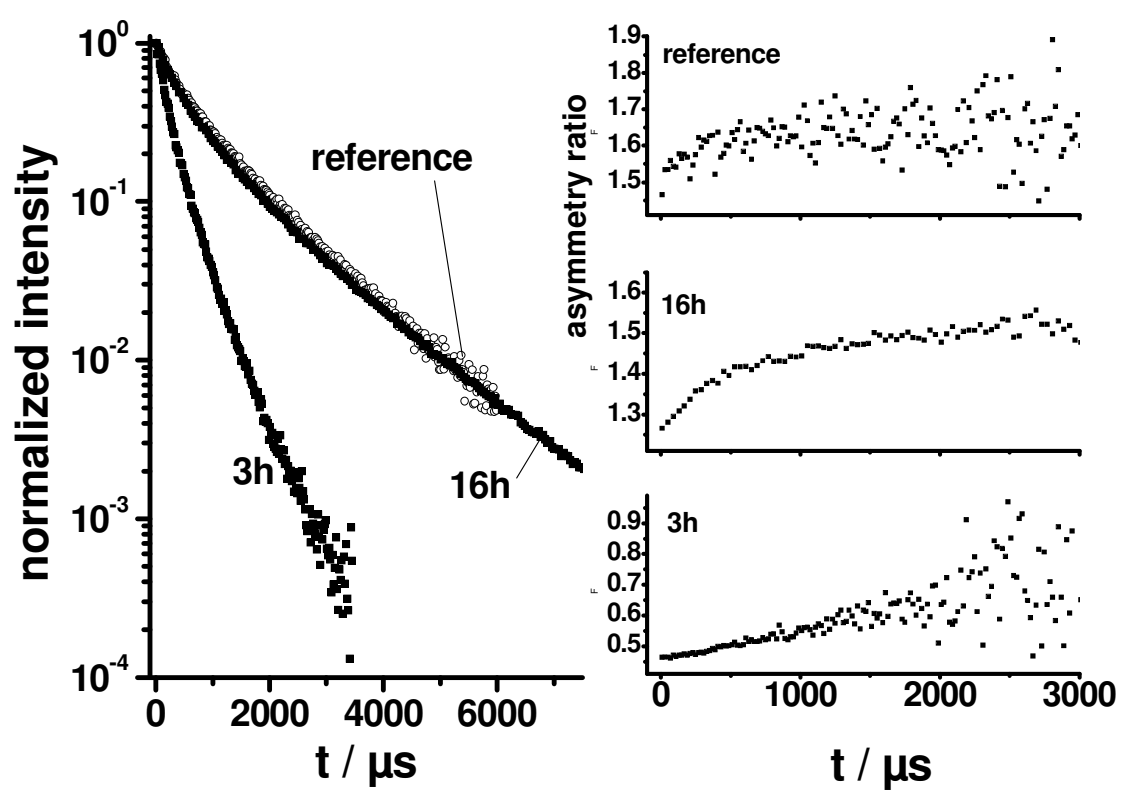

Figure 8 Luminescence decay at $\lambda_{\mathrm{em}}=395 \mathrm{~nm}$ (left) and time-resolved asymmetry ratio (right) of the different samples $\left(\lambda_{\mathrm{ex}}=395 \mathrm{~nm}\right)$ in water suspension. The asymmetry ratio is calculated as the intensity ratio of the ${ }^{5} D_{0}{ }^{-} F_{1}$ and the ${ }^{5} D_{0}{ }^{7} F_{2}$ transition at $\lambda_{\mathrm{em}}=595 \mathrm{~nm}$ and $615 \mathrm{~nm}$, respectively. 
In the luminescence decays, quenching contributions from ligands are seen. The luminescence decays of all samples are multiexponential, which may be connected to different species in the sample (e.g., surface and bulk europium ions interacting differently with surrounding water molecules, which are strong luminescence quenchers due to the effective coupling to $\mathrm{OH}$ vibrations). The presence of two species (bulk and surface) can be seen in the timedependence of the asymmetry ratio of the reference and the sample isolated after $16 \mathrm{~h}$, which both approach a plateau at long times after the laser flash (right side of Figure 8, top and middle $)^{[29]}$. Striking is the fast (compared to the other samples) luminescence decay of the mesocrystal sample isolated after $3 \mathrm{~h}$, which may be attributed to a strong coupling to the presence of the polymer gel matrix and the subsequent efficient luminescence quenching (e.g, via matrix vibrations).

The whole reaction pathway reminds of other, related observations of mesocrystal formation via crystallisation in a gel-template. One of the best investigated examples of mesocrystal development in a gel is the formation of the hexagonal-prismatic seed crystal of fluorapatite in gelatin ${ }^{[30]}$. The crystallization in a gel is very suited for the formation of mesocrystals as the crystal growth takes place at a high level of supersaturation and in the absence of flow and mechanical distortion ${ }^{[31]}$. This results on the one hand in an increase of the nucleation of small nanoparticles, which in addition are able to interact unperturbed and to arrange. Furthermore, the reduced diffusion in the gel ensures sufficiently slow growth ${ }^{[31]}$. The level of supersaturation of $\mathrm{Eu}^{3+}$-ions along the Europium-ACUSOL-gel is very high and nucleation occurs since the Accusol gel acts as an "ion sponge". Small EuF $F_{3}$ nanoparticles develop in the polymer gel, which then form the mesocrystals. Since $\mathrm{EuF}_{3}$ has a hexagonal crystal structure, it has by symmetry no dipole moment, which could generate the mutual alignment of the nanocrystals in the mesocrystal. However, $\mathrm{EuF}_{3}$ has polar faces, namely $\{001\},\{110\}$ and \{011\} (see Figures SI 3-5), which can be Europium-terminated and therefore strongly positive. This is favourable for the adsorption of the negatively charged copolymer. If a 
particle is nucleated in the vicinity of the polymer, the polymer can adsorb on one of these charged faces and block them from further growth. However, adsorption can - for energetic reasons- only occur on one side of a particle, thus creating two oppositely charged faces, as was already discussed earlier for $\mathrm{CaCO}_{3}{ }^{[12]}$ and $\mathrm{BaCrO}_{4}{ }^{[32]}$ This leads to the formation of a strong dipole, resulting in the mutual alignment of adjacent nanoparticles. We propose this mechanism also to hold true for the formation of the $\mathrm{EuF}_{3}$ mesocrystals in the copolymer gel. It is interesting to note that our case is different from the fluoroapatite mesocrystals formed in a gelatin gel ${ }^{[33]}$. Gelatin triple helices carry a dipole moment, which generates the intrinsic electric field aligning the nanoparticles. In the present example, this cannot be the case, and dipole fields can only be generated by unsymmetrical polymer adsorption.

\section{CONCLUSIONS}

In conclusion we have succeeded in fabrication of Europium(III)fluoride mesocrystals by a simple reaction in an organic matrix. It was demonstrated that $\mathrm{Eu}^{3+}$-ions react with the polymer ACUSOL 588G to a gel. Adsorption of the polyanion on a charged face of a particle nucleated in the vicinity of the polymer leads to oppositely charged faces resulting in a dipole moment. Due to this dipole field, the arrangement of the particles takes place according to the particular dipole-dipole-interactions. This is supported by the luminescence results, which confirm the presence of a modified Eu-ion surrounding in presence of the polymer gel matrix. The polymer gel has multiple roles in this complex structuration process. It acts as an ion sponge that generates the high supersaturation in the gel phase leading to particle nucleation in the gel only. Secondly, it leads to the formation of a dipole field by adsorption onto a charged polymer face in the vicinity of the gel. In the present case, the mesocrystal gel structure is however only transient. With time, the gel dissolves again after the crosslinking Europium-ions were consumed for particle growth. Our example shows that complex 
functional mesocrystal structures can be assembled in very simple self-organized reaction schemes.

Nanostructured materials have received extensive attention due to their unusual properties and potential applications. For example, Europium(III)fluoride is used for high-performance luminescence displays or for laser materials. The here shown mesocrystaline material might be advantageous for these applications since a mesocrystal combines high crystalinity with a small particle size.

\section{EXPERIMENTAL SECTION}

Materials: Sodium fluoroborate $\left(\mathrm{NaBF}_{4}\right.$, Fluka, $\left.\geq 98 \%\right)$, Europium(III) nitrate pentahydrate $\left(\mathrm{Eu}\left(\mathrm{NO}_{3}\right)_{3} \cdot 5 \mathrm{H}_{2} \mathrm{O}\right.$, Aldrich, 99.9\%) and the Polymer ACUSOL 588G (Acrylic acid / sulfonic acid copolymer, likely with 2-acrylamido-2-methylpropanesulfonic acid, AMPS content from elemental analysis $=14.7$ mol- $\%, M_{\mathrm{w}}=12000 \mathrm{~g} / \mathrm{mol}$, a well known antiscale additive donated by Benckiser, were used without further purification.

Synthesis: $25 \mathrm{~mL}$ of ACUSOL aqueous solution $(\mathrm{c}=0.4 \mathrm{~g} / \mathrm{L})$ were mixed with $25 \mathrm{~mL}$ of $\mathrm{NaBF}_{4}$ aqueous solution $(\mathrm{c}=160 \mathrm{mM})$ under stirring in a beaker. After a few minutes $50 \mathrm{~mL}$ of $\mathrm{Eu}\left(\mathrm{NO}_{3}\right)_{3} \cdot 5 \mathrm{H}_{2} \mathrm{O}$ aqueous solution $(\mathrm{c}=20 \mathrm{mM})$ were added. The mixture became turbid immediately. The reaction mixture was stirred at room temperature for $3 \mathrm{~h}$ respectively $16 \mathrm{~h}$.

Afterwards the supernatant solution was removed by centrifugation. The precipitate was washed with distilled water several times as well as twice with ethanol via centrifugation.

Between the centrifugation steps, the sample was treated by ultrasonics for a few minutes to dissolve the precipitate in water. The final product was dried at $70^{\circ} \mathrm{C}$ under vacuum over night.

Characterisation: The crystals were characterized with different microscope techniques. The light microscope images were obtained with a Leica microscope type DMR B with and without crossed polarisers. The microstructure was also analyzed by a scanning electron 
microscope (SEM) LEO 1550-Gemini-System. The transmission electron microscope (TEM) images were recorded on a Zeiss EM $912 \Omega$ microscope operated at $120 \mathrm{kV}$.

The XRD data were measured in reflection mode $\left(\mathrm{Cu}-\mathrm{K}_{\alpha}\right.$ radiation $)$ on a Bruker $\mathrm{D} 8$ diffractometer equipped with scintillation counter.

The luminescence of the crystals was measured using combined Nd:YAG OPO laser (QuantaRay, Spectra Physics) OPO system operated at $20 \mathrm{~Hz}$ (versaScan, GWU Lasertechnik) for excitation at $\lambda_{\mathrm{ex}}=395 \mathrm{~nm}$. The emission of the samples was detected in the wavelength range of $550 \mathrm{~nm}<\lambda_{\mathrm{em}}<800 \mathrm{~nm}$ by a spectrograph (MS257, Oriel Instruments) equipped with an iCCD camera (DH720-18H-13, Andor Technology). In the luminescence measurements timeresolved emission spectra (TRES) were recorded based on a gated (box-car) detection scheme. In a typical experiment the luminescence signal was integrated for a gate time $\Delta \mathrm{t}=$ $100 \mu \mathrm{s}$ at variable gate step $\delta \mathrm{t}$ after the laser pulse in the time range of $10 \mu \mathrm{s}<\delta \mathrm{t}<5000 \mu \mathrm{s}$ with a gate step of $40 \mu$ s. The luminescence measurements were performed on suspensions of the samples at room temperature.

\section{ACKNOWLEDGEMENT}

We thank Rona Pitschke and Heike Runge for SEM- and TEM- measurements as well as Christina Diehl and Ina Dambowsky for discussions about the chemical structure of the polymer, Peter Cernoch for DLS-Measurements, and Katlen Brennenstuhl for the measurement of the TRES. The Max-Planck-society is acknowledged for financial support.

\section{REFERENCES}

[1] H. Cölfen, M. Antonietti, Angew. Chem.-Int. Ed. 2005, 44, 5576.

[2] H. A. Cölfen, M., Mesocrystals and Non Classical Crystallization, Wiley, 2008.

[3] L. Zhou, P. O'Brien, Small 2008, 4, 1566.

[4] L. Zhou, D. S. Boyle, P. O'Brien, Chem. Comm. 2007, 144.

[5] L. Zhou, D. S. Boyle, P. O'Brien, J. Am. Chem. Soc. 2008, 130, 1309. 
[6] J. P. Ge, Y. X. Hu, M. Biasini, W. P. Beyermann, Y. D. Yin, Angew. Chem.-Int. Ed. 2007, 46, 4342.

[7] N. G. Zhuravleva, A. A. Eliseev, N. A. Sapoletova, A. V. Lukashin, U. Kynast, Y. D. Tretyakov, Mat. Sci. Eng. C-Bio. S 2005, 25, 549.

[8] A. Braud, S. Girard, J. L. Doualan, M. Thuau, R. Moncorge, A. M. Tkachuk, Phys. Rev. B 2000, 61, 5280.

[9] K. Kömpe, H. Borchert, J. Storz, A. Lobo, S. Adam, T. Moller, M. Haase, Angew. Chem.-Int. Ed. 2003, 42, 5513.

[10] J. W. Stouwdam, F. van Veggel, Nano Lett. 2002, $2,733$.

[11] R. X. Yan, Y. D. Li, Adv. Funct. Mater. 2005, 15, 763.

[12] M. Wang, Q. L. Huang, J. M. Hong, X. T. Chen, Z. L. Xue, Cryst. Growth Des. 2006, $6,2169$.

[13] M. Wang, Q. L. Huang, J. M. Hong, X. T. Chen, Z. L. Xue, Cryst. Growth Des. 2006, 6, 1972.

[14] T. Cui, Zhang,H., Du,N., Chen,B., Li,D., Yang,D., Mater. Chem. Phys. 2009, 115, 562.

[15] Z. Miao, Liu,Z., Ding,K., Han,B., Miao,S., Nanotechnol. 2007, 18.

[16] C. K. Jayasankar, Phys. Stat. sol. 1985, 133.

[17] S. Busch, H. Dolhaine, A. DuChesne, S. Heinz, O. Hochrein, F. Laeri, O. Podebrad, U. Vietze, T. Weiland, R. Kniep, Eur. J. Inorg. Chem. 1999, 1643.

[18] R. Kniep, S. Busch, Angew. Chem.-Int. Ed. 1996, 35, 2624.

[19] M. Niederberger, H. Cölfen, Phys. Chem. Chem. Phys. 2006, 8, 3271.

[20] R. Q. Song, H. Cölfen, Adv. Mater. 2009, 21, 1.

[21] T. Kato, A. Sugawara, N. Hosoda, Adv. Mater. 2002, 14, 869.

[22] T. Nishimura, T. Ito, Y. Yamamoto, M. Yoshio, T. Kato, Angew. Chem.-Int. Ed. 2008, 47, 2800.

[23] T. Sakamoto, A. Oichi, Y. Oaki, T. Nishimura, A. Sugawara, T. Kato, Cryst. Growth Des. 2009, 9, 622.

[24] Y. Yamamoto, T. Nishimura, A. Sugawara, H. Inoue, H. Nagasawa, T. Kato, Cryst. Growth Des. 2008, 8, 4062.

[25] A. Sugawara, T. Nishimura, Y. Yamamoto, H. Inoue, H. Nagasawa, T. Kato, Angew. Chem.-Int. Ed. 2006, 45, 2876.

[26] A. M. Belcher, X. H. Wu, R. J. Christensen, P. K. Hansma, G. D. Stucky, D. E. Morse, Nature 1996, 381, 56.

[27] N. Nassif, N. Pinna, N. Gehrke, M. Antonietti, C. Jager, H. Cölfen, PNAS 2005, 102, 12653.

[28] B. Marmodée, J. S. de Klerk, F. Ariese, C. Gooijer, M. U. Kumke, Anal. Chim. Acta 2009, 652, 285.

[29] C. Tiseanu, M. U. Kumke, V. I. Parvulescu, J. Martens, J. Appl. Phys. 2009, 105, 063521.

[30] P. Simon, U. Schwarz, R. Kniep, Journal of Materials Chemistry 2005, 15, 4992.

[31] A. Putnis, M. Prieto, L. Fernandezdiaz, Geol. Mag. 1995, 132, 1.

[32] S. H. Yu, H. Cölfen, M. Antonietti, Chem Eur J 2002, 8, 2937.

[33] P. Simon, D. Zahn, H. Lichte, M. Kniep, Angew. Chem.-Int. Ed. 2006, 45, 1911. 